\title{
Probing the tricritical endpoint of QCD phase diagram at NICA- FAIR energies
}

\author{
K. A. Bugaev ${ }^{1}$, A. I. Ivanytskyi ${ }^{1}$, V. V. Sagun ${ }^{1,2}$, G. M. Zinovjev ${ }^{1}$, E. G. Nikonov ${ }^{3}$, R. Emaus ${ }^{4}$, L. V. \\ Bravina $^{4}$, E. E. Zabrodin ${ }^{4,5,6}$, and A. V. Taranenko ${ }^{6}$ \\ ${ }^{1}$ Bogolyubov Institute for Theoretical Physics of the National Academy of Sciences of Ukraine, 03680 Kiev, \\ Ukraine \\ ${ }^{2}$ Centro de Astrofísica e Gravitação - CENTRA, Departamento de Física, Instituto Superior Técnico, Univer- \\ sidade de Lisboa, 1049-001 Lisboa, Portugal \\ ${ }^{3}$ Laboratory for Information Technologies, Joint Institute for Nuclear Research, Dubna 141980, Russia \\ ${ }^{4}$ Department of Physics, University of Oslo, PB 1048 Blindern, N-0316 Oslo, Norway \\ ${ }^{5}$ Skobeltzyn Institute of Nuclear Physics, Moscow State University, 119899 Moscow, Russia \\ ${ }^{6}$ National Research Nuclear University "MEPhl" (Moscow Engineering Physics Institute), 115409 Moscow, \\ Russia
}

\begin{abstract}
In this contributions we discuss the novel version of hadron resonance gas model which is based on the induced surface tension concept. Also we present new arguments in favor of a hypothesis that the chiral symmetry restoration transition in central nuclear collisions may occur at the center of mass energies $4.3-4.9 \mathrm{GeV}$ and that the deconfinement phase transition may occur at the center of mass energies $8.8-9.2 \mathrm{GeV}$. These arguments are based on the unique thermostatic properties of the mixed phase and the ones of an exponential mass spectrum of hadrons.
\end{abstract}

\section{Introduction}

During more than thirty years of searches for the deconfinement and for the chiral symmetry restoration (CSR) phase transitions (PT) in heavy ion collisions (HIC) there were made many interesting discoveries [1], but until recently the situation with the reliable signals was rather controversial. Although such peculiar irregularities in excitation functions of experimental data, known in the literature as the Kink [2], the Strangeness Horn [3] and the Step [4], motivated the experimental program at CERN SPS to lower the collision energy in order to locate the onset of deconfinement, an absence of firm theoretical explanation of these irregularities was always the source of natural doubts about their relation to deconfinement PT. After a discovery of the step-like structure of the inverse slope parameter of transverse momentum spectra of $\mathrm{K}^{+}$and $\mathrm{K}^{-}$mesons in proton-proton collisions [5] at the same collision energies as in HIC, the whole logic of claims made in Ref. [6] was destroyed. Indeed, the major argument of Ref. [6] that the Kink, Strangeness Horn and Step observed in HIC are the signals of deconfinement is based on the fact that the corresponding quantities observed in proton-proton collisions behave entirely different. Therefore, after the similarity of transverse momentum spectra of $\mathrm{K}^{+}$and $\mathrm{K}^{-}$mesons measured in $\mathrm{HIC}$ and in proton-proton collision was found, it became evident that 
without firm theoretical back-up all speculations of Ref. [6] about the Kink, Strangeness Horn and Step as the signals of the onset of deconfinement are not trustworthy.

It is necessary to stress that such a situation is not surprising, since in HIC we are dealing with finite systems for which there is no rigorous theory of first order PT of the liquid-gas type. Although some progress in formulation of the finite volume analog of phases for liquid-gas PT in finite static systems was achieved on the basis of exactly solvable models $[7,8]$, presently it is unclear how to apply these results to the fast expanding systems created in HIC. It is, therefore, clear that any new signal of the first order liquid-gas PT which can be applied to finite systems is very important for a completion of the HIC programs which are performed presently at SPS CERN, LHC CERN and RHIC BNL and which are planned for the nearest future at NICA JINR and FAIR GSI. Therefore, here we would like to present a few irregularities found during last few years [9-13] in symmetric $\mathrm{HIC}$ at chemical freeze-out (CFO) and to briefly discuss their relation to deconfinement PT and to CSR PT.

The work is organized as follows. In Sect. 2 we remind the basic elements of the newest hadron resonance gas model (HRGM) [14-16] based on the concept of induced surface tension [17]. The new signals of CSR and deconfinement PT are discussed in Sect. 3, whereas our conclusions are summarized in Sect. 4.

\section{HRGM with multicomponent hard-core repulsion}

Traditionally, the HRGM [18-24] is used to determine the parameters of CFO from the measured hadronic yields. Presently its version with the multicomponent hard-core repulsion between hadrons [14, 19-23] provides the best description of all independent hadronic multiplicity rations measured in the central HIC at the center of mass energies $\sqrt{s_{N N}}=$ 2.7, 3.1, 3.8, 4.3, 4.9, 6.3, 7.6, 8.8, 9.2, 12.3, 17.3, 62.4, 130, 200, $2760 \mathrm{GeV}$. There are three main reasons to employ the HRGM as the equation of state (EoS) of hadronic matter. First, it is well known that for temperatures below $170 \mathrm{MeV}$ and low baryonic charge densities the mixture of stable hadrons and their resonances whose interaction is taken into account by the quantum second virial coefficients behaves as the mixture of nearly ideal gases of stable particles which, however, includes both the hadrons and their resonances, but taken with their averaged masses [25]. As it was shown in Ref. [25], the main reason for such a behavior is related to an almost complete cancellation between the attraction and repulsion contributions in the quantum second virial coefficients. Therefore, the remaining deviation from the ideal gas (a weak repulsion) is usually described by the classical second virial coefficients. The second reason to use the HRGM as the hadronic matter EoS is that in this case its pressure will not exceed the one of quark-gluon plasma which, nevertheless, may happen, if the hadrons are treated as the mixture of ideal gases $[14,26]$. Finally, the third reason to employ the HRGM is the practical one: due to the fact that hard-core repulsion is the contact interaction, the energy per particle of such an EoS coincides with the one of an ideal gas, even for quantum particles [16]. As a consequence, during the subsequent evolution of the system after the CFO to the kinetic freeze-out $[27,28]$ one should not somehow "convert" the potential energy of interacting particles into their kinetic energy and/or into the masses of newly born particles. These reasons allow one not only to consider the HRGM as an extension of the famous statistical bootstrap model [29] supplemented by the hard-core repulsion which, in addition, has a truncated hadronic mass spectrum, but to effectively use it to describe the hadronic multiplicities measured in the HIC experiments.

Despite many valuable results obtained with the HRGM during last years, the hard-core radii are well established at the moment for the most abundant hadrons only, namely for pions $\left(R_{\pi} \simeq 0.15\right.$ $\mathrm{fm})$, for lightest $\mathrm{K}^{ \pm}$-mesons $\left(R_{K} \simeq 0.395 \mathrm{fm}\right)$, for nucleons $\left(R_{p} \simeq 0.365 \mathrm{fm}\right)$ and for lightest (anti) $\Lambda$ hyperons $\left(R_{\Lambda} \simeq 0.085 \mathrm{fm}\right)[14,15]$. Nevertheless, we hope that the new data of high quality which are 
expected to be measured during the Beam Energy Scan II at RHIC BNL (Brookhaven) [30], and at the accelerators of new generation, i.e. at NICA JINR (Dubna) [31] and FAIR GSI (Darmstadt) [32], will help us to determine the hard-core radii of other measured hadrons with high accuracy. However, the traditional multicomponent HRGM is not suited for such a purpose, since for $N$ different hard-core radii it is necessary to find a solution of $N$ transcendental equations. Hence, a further increase of the number of hard-core radii (i.e., $N \sim 100$, corresponding to the various hadronic species created in a collision) will lead to a huge increase of computational time and in this way it will destroy the main attractive feature of the HRGM, i.e., its simplicity. To overcome this problem the novel HRGM based one the concept of the induced surface tension (IST) [17] was recently developed in Refs. [14-16].

It is a system of coupled equations for the pressure $p$ and the induced surface tension coefficient $\Sigma$

$$
\begin{aligned}
p & =\sum_{k=1}^{N} p_{k}=T \sum_{k=1}^{N} \phi_{k} \exp \left[\frac{\mu_{k}}{T}-\frac{4}{3} \pi R_{k}^{3} \frac{p}{T}-4 \pi R_{k}^{2} \frac{\Sigma}{T}\right], \\
\Sigma & =\sum_{k=1}^{N} \Sigma_{k}=T \sum_{k=1}^{N} R_{k} \phi_{k} \exp \left[\frac{\mu_{k}}{T}-\frac{4}{3} \pi R_{k}^{3} \frac{p}{T}-4 \pi R_{k}^{2} \alpha \frac{\Sigma}{T}\right], \\
\mu_{k} & =\mu_{B} B_{k}+\mu_{I 3} I_{3 k}+\mu_{S} S_{k},
\end{aligned}
$$

where $\alpha=1.245$, and $\mu_{B}, \mu_{S}, \mu_{I 3}$ are the baryonic, the strangeness, and the third projection of the isospin chemical potential, respectively. Here $B_{k}, S_{k}, I_{3 k}, m_{k}$ and $R_{k}$ denote, respectively, the corresponding charges, mass, and hard-core radius of the $k$-th hadronic species. The sums in Eqs. (1) and (2) run over all hadronic species; their corresponding antiparticles are considered as independent species and, hence, $p_{k}$ and $\Sigma_{k}$ are, respectively, the partial pressure and the partial induced surface tension coefficient of the $k$-th hadronic species.

The one-particle thermal density $\phi_{k}$ in Eqs. (1) and (2) accounts for the Breit-Wigner mass attenuation and is written in the Boltzmann approximation (the quantum one is given in [16])

$$
\phi_{k}=g_{k} \gamma_{S}^{\left|s_{k}\right|} \int_{M_{k}^{T h}}^{\infty} \frac{d m}{N_{k}\left(M_{k}^{T h}\right)} \frac{\Gamma_{k}}{\left(m-m_{k}\right)^{2}+\Gamma_{k}^{2} / 4} \int \frac{d^{3} p}{(2 \pi)^{3}} \exp \left[-\frac{\sqrt{p^{2}+m^{2}}}{T}\right]
$$

where $g_{k}$ is the degeneracy factor of the $k$-th hadronic species, $\gamma_{S}$ is the strangeness suppression factor [33], $\left|s_{k}\right|$ is the number of valence strange quarks and antiquarks in this hadron species, $N_{k}\left(M_{k}^{T h}\right) \equiv$ $\int_{M_{k}^{T h}}^{\infty} \frac{d m \Gamma_{k}}{\left(m-m_{k}\right)^{2}+\Gamma_{k}^{2} / 4}$ denotes a normalization factor, while $M_{k}^{T h}$ corresponds to the decay threshold mass of the $k$-th hadronic species and $\Gamma_{k}$ denotes its width.

To apply the system of Eqs. (1), (2), and (3) to study nuclear collisions it should be supplemented by the strange charge conservation law, which is equivalent to a vanishing net strangeness density

$$
\rho_{S} \equiv \frac{\partial p}{\partial \mu_{S}}=\sum_{k} S_{k} \rho_{k}=0
$$


where $\rho_{k}$ is density of hadrons of sort $k$ given by the system of equations

$$
\begin{aligned}
& \rho_{k} \equiv \frac{\partial p}{\partial \mu_{k}}=\frac{1}{T} \cdot \frac{p_{k} a_{22}-\Sigma_{k} a_{12}}{a_{11} a_{22}-a_{12} a_{21}}, \\
& a_{11}=1+\frac{4}{3} \pi \sum_{k} R_{k}^{3} \frac{p_{k}}{T}, \quad a_{12}=4 \pi \sum_{k} R_{k}^{2} \frac{p_{k}}{T}, \\
& a_{22}=1+4 \pi \alpha \sum_{k} R_{k}^{2} \frac{\Sigma_{k}}{T}, \quad a_{21}=\frac{4}{3} \pi \sum_{k} R_{k}^{3} \frac{\Sigma_{k}}{T} .
\end{aligned}
$$

Note that in contrast to the usual multicomponent HRGM formulations to determine the particle number densities $\left\{\rho_{k}\right\}$ one needs to solve only a system of three equations, namely Eqs. (1), (2) and (5), irrespective to the number of different hard-core radii in the model. Therefore, we believe that IST EoS defined by system (1)-(5) is perfectly suited for the analysis of all hadronic multiplicities which will be measured in the nearest future at RHIC, NICA and FAIR.

Another great advantage of the IST EoS is its validity up to the packing fractions $\eta \equiv \sum_{k} \frac{4}{3} \pi R_{k}^{3} \rho_{k} \simeq$ $0.2-0.22$ [14-16], i.e., at the particle number densities for which the traditional HRGM based on the Van der Waals approximation [18-23] is absolutely incorrect.

Using the particle number density (6) of $k$-th sort of hadrons one can determine the thermal $N_{k}^{\text {th }}=$ $V \rho_{k}$ ( $V$ is the effective volume at CFO) and the total multiplicities $N_{k}^{t o t}$. The latter should account for the hadronic decays after the $\mathrm{CFO}$ and then the ratio of total hadronic multiplicities becomes

$$
\frac{N_{k}^{t o t}}{N_{j}^{t o t}}=\frac{\rho_{k}+\sum_{l \neq k} \rho_{l} B r_{l \rightarrow k}}{\rho_{j}+\sum_{l \neq j} \rho_{l} B r_{l \rightarrow j}},
$$

where $B r_{l \rightarrow k}$ is the branching ratio, i.e., a probability of particle $l$ to decay strongly into a particle $k$. More details on the fitting procedure of experimental data with the HRGM can be found in [22].

\section{New Signals of QCD Phase Transitions}

Using the multicomponent HRGM it was possible to reveal a few irregularities of thermodynamic quantities observed at CFO and to relate them to two QCD phase transitions. The most remarkable irregularities include two sets of correlated quasi-plateaus found in [9-11] which are located at the collision energy ranges $\sqrt{s_{N N}} \simeq 3.8-4.9 \mathrm{GeV}$ and $\sqrt{s_{N N}} \simeq 7.6-9.2 \mathrm{GeV}$, and two peaks of trace anomaly $\delta=\frac{(\epsilon-3 p)}{T^{4}}$ (here $\epsilon, p$ and $T$ denote, respectively, the energy density of the system, its pressure and temperature) observed at the maximal energy of each set of quasi-plateaus $[11,12]$. Figure 1 shows the trace anomaly $\delta$ as a function of center-of-mass collision energy and as a function of the CFO temperature obtained by the IST EoS [12]. As one can see from Fig. 2 the baryonic charge density at CFO exhibits two sharp peaks which are located exactly at the collision energies of the trace anomaly peaks [12], i.e. at $\sqrt{s_{N N}}=4.9 \mathrm{GeV}$ and $\sqrt{s_{N N}}=9.2 \mathrm{GeV}$.

Although the set of low energy quasi-plateaus was predicted a long time ago $[34,35]$ as a manifestation of the anomalous thermodynamic properties of quark-gluon-hadron mixed phase, the interpretation of the high energy set of quasi-plateaus was difficult, since the generalized shock adiabat model $[34,35]$ which predicted such quasi-plateaus can be applied to the collision energies $\sqrt{s_{N N}}>8 \mathrm{GeV}$ qualitatively only. Thus, the first hints in favor of two QCD phase transitions were found in 2014 in [9], but the self-consistent interpretation of these irregularities was worked out only in 2017 in [12]. This success was achieved after finding out in Ref. [12] the number of effective degrees of freedom of the phase formed at the collision energies $\sqrt{s_{N N}}=4.9-9.2 \mathrm{GeV}$ and after explaining in Ref. [12] 

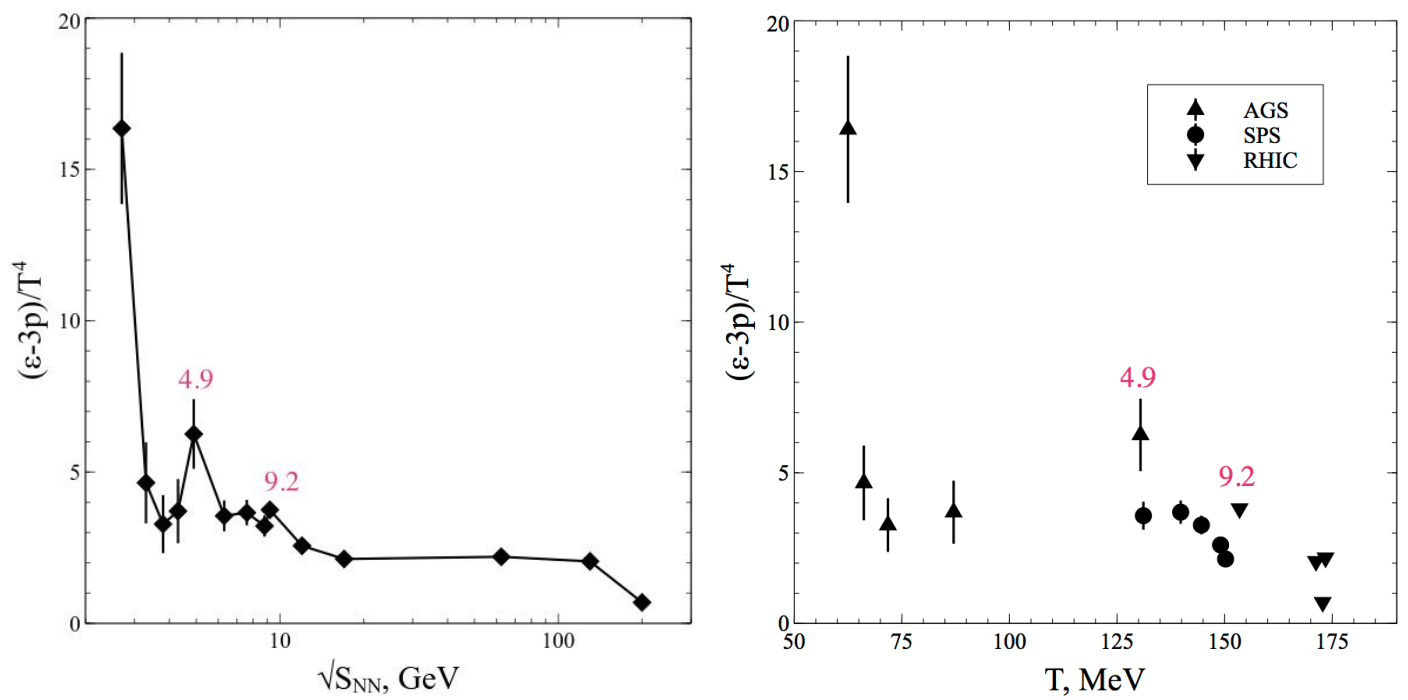

Figure 1. Trace anomaly dependence on the center of mass collision energy (left) and on the CFO temperature (right). The numbers above the symbols correspond to a particular value of center of mass collision energy. In the right panel the points $\sqrt{s_{N N}}=4.9 \mathrm{GeV}$ and $\sqrt{s_{N N}}=9.2 \mathrm{GeV}$ are well separated from the neighboring ones.

the peculiar collision energy dependence of the strangeness enhancement factor $\gamma_{s}$. Since the number of effective degrees of freedom was already discussed in Ref. [13], here we discuss the novel signals of two QCD phase transitions related to the $\gamma_{s}$ factor.

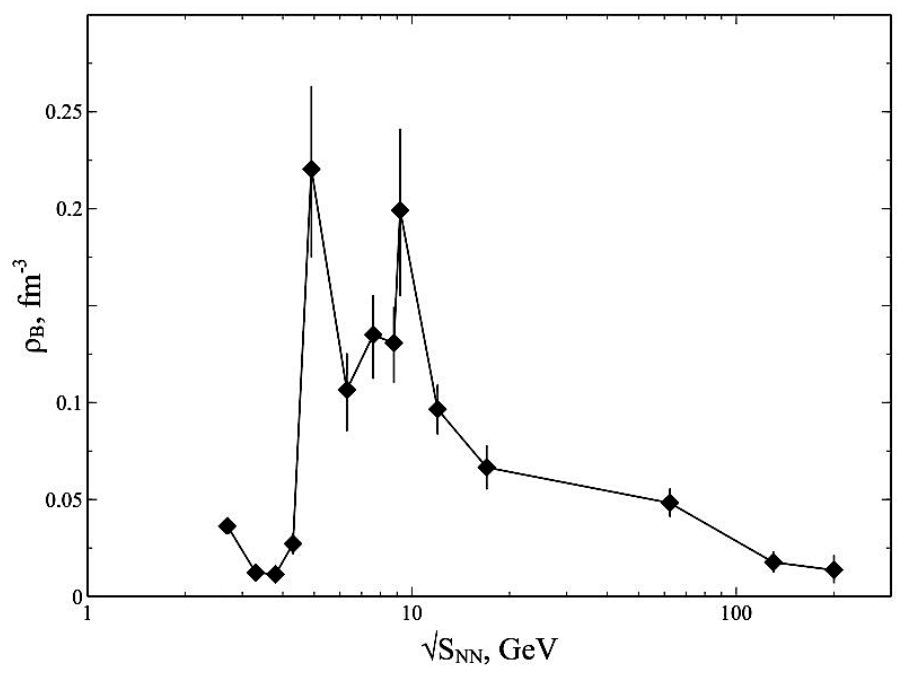

Figure 2. Collision energy dependence of the baryonic charge density found by the IST EoS at CFO. 

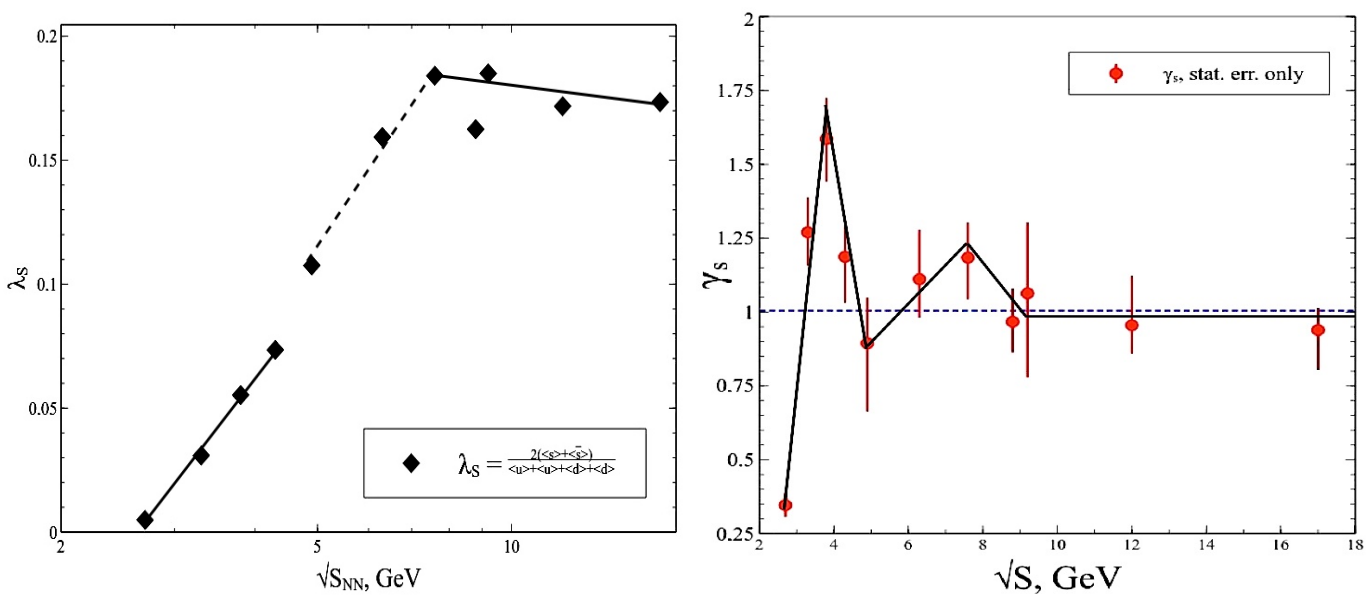

Figure 3. Comparison of the collision energy dependence of the modified Wroblewski factor $\lambda_{s}$ (left panel) and the strangeness suppression factor $\gamma_{s}$ (right panel).

For more than three decades the HIC community is discussing the relation of strange charge irregularities to a deconfinement PT, but until now there is no complete understanding of such a relation. To demonstrate a single aspect of this problem, let us compare the left and the right panels of Fig. 3 . From the left panel of Fig. 3 one can see that up to the collision energy $\sqrt{s}_{N N}=8.8 \mathrm{GeV}$ there is a monotonic increase of the modified Wroblewski factor $\lambda_{s}$ [36] which was defined in Ref. [12] as

$$
\lambda_{s} \equiv \frac{2 \sum_{n}\left(N_{n}^{S}+N_{n}^{\bar{S}}\right) \rho_{n}}{\sum_{n}\left(N_{n}^{u}+N_{n}^{\bar{u}}+N_{n}^{d}+N_{n}^{\bar{d}}\right) \rho_{n}},
$$

where in the numerator $N_{n}^{S}$ and $N_{n}^{\bar{S}}$ denote, respectively, the number of strange valence quarks and antiquarks in the hadron of sort $n$, whereas $N_{n}^{u}$ and $N_{n}^{d}$ in the denominator denote, respectively, the number of $u$ and $d$ valence quarks in it (for antiquarks we used $N_{n}^{\bar{u}}$ and $N_{n}^{\bar{d}}$ ). Note that the denominator in Eq. (10) differs from the traditional Wroblewski factor [36] because it accounts for the number of $u$ and $d$ valence quarks and antiquarks, and not only the ones which are paired to their valence antiquarks (quarks). This quantity is more convenient and has a simple physical meaning: $\lambda_{s}(10)$ can be treated as an asymmetry of the strange (anti)quark production compared to the $u$ and $d$ quarks. Also, the $\lambda_{s}$ factor is directly related to the concentration of (anti)strange quarks in the system, i.e

$$
v_{s}=\frac{\sum_{n}\left(N_{n}^{S}+N_{n}^{\bar{S}}\right) \rho_{n}}{\sum_{n}\left(N_{n}^{u}+N_{n}^{\bar{u}}+N_{n}^{d}+N_{n}^{\bar{d}}+N_{n}^{S}+N_{n}^{\bar{S}}\right) \rho_{n}} \equiv \frac{\lambda_{s}}{2+\lambda_{s}} .
$$

On the other hand the right panel of Fig. 3 demonstrates a very complicated behavior of the $\gamma_{s}$ factor right for collision energies $\sqrt{s}_{N N}<8.8 \mathrm{GeV}$, while for $\sqrt{s}_{N N} \geq 8.8 \mathrm{GeV}$ the strangeness demonstrates a full chemical equilibrium, i.e., $\gamma_{s}=1$. Such a behavior was first found in 2013, when the $\gamma_{s}$ factor was used in the multicomponent HRGM of Ref. [21], but until recently its reason for such a behavior remained a hard puzzle. First we discuss the collision energies above $8.8 \mathrm{GeV}$. As it is 
argued in [12] the fact that for these energies $\gamma_{s}=1$ within the error bars can be naturally explained by the formation of the quark-gluon bags with the exponential mass spectrum proposed by R. Hagedorn [29]. As it was predicted in [37] and later on shown numerically in [38, 39], the exponential mass spectrum acts as a perfect thermostat and a perfect particle reservoir, i.e., all particles which appear from such a bag at its hadronization will inevitably be born in a state of full thermal and chemical equilibrium with it [37-39]. Therefore, most probably, the collision energy $\sqrt{s}_{N N} \simeq 8.8-9.2 \mathrm{GeV}$ corresponds to the deconfinement PT to QGP $[12,13]$. More arguments in favor of this conclusion are given in $[12,13]$.

As argued in [12] the dale of $\gamma_{s}$ factor, i.e., $\gamma_{s}=1$ at $\sqrt{s}_{N N}=4.9 \mathrm{GeV}$, is directly related to the 1-st order PT. Indeed, in Ref. [37] a few examples of the explicit thermostats and explicit particle reservoirs are discussed and it is argued that under the constant pressure condition the mixed phase of the 1-st order PT, i.e., two pure phases being in a full thermal and chemical equilibrium with each other, represent both a thermostat and a particle reservoir as long as there is sufficient energy to keep a constant temperature. This means that under the constant pressure condition an explicit thermostat keeps a constant temperature despite imparting out (in) some amount of heat. The heat transmission only changes the volume fractions of two phases: the phase with higher heat capacity (for definiteness, a liquid) condenses a certain amount of gas under external cooling or it partly evaporates some amount of gas under external heating. Evidently, for finite systems the amount of transmitted heat is also finite and it depends on the masses of both phases and their heat capacities. Similarly, one can add to or remove from the system some amount of each phase, but under the constant pressure condition the system (mixed phase) will maintain a constant temperature and a full chemical equilibrium, i.e. the equal values of chemical potentials for both pure phases being in contact. Therefore, up to some maximal value any amount of the removed phase, namely the gas of hadrons, for definiteness, will be in a full chemical and thermal equilibrium with the mixed phase. Apparently, the maximal amount of removed phase depends on the masses of both phases and their energy densities. However, to justify such arguments it is necessary to assume that the mixed phase has sufficiently large volume, so the finite size effects are not important.

The generalized shock adiabat model of central HIC [9-11, 34, 35] allows one to reliably determine the initial conditions for the subsequent hydrodynamic evolution. As it was shown numerically in Refs. [9-11] the initial states which correspond to a mixed phase have practically the same pressure and, hence, the condition of constant pressure is valid with the accuracy of about two percent [12]. Therefore, the strangeness equilibrium dale seen at the collision energy $\sqrt{s}_{N N}=4.9 \mathrm{GeV}$ is a new and an independent signal of the mixed phase formation in HIC. Since the number of the effective degrees of freedom of almost massless particles estimated in [12] is about 1770, it evidences for the CSR PT in hadronic phase at the collision energies $\sqrt{s}_{N N}=4.3-4.9 \mathrm{GeV}$. Furthermore, since above we came to a conclusion that at $\sqrt{s}_{N N}=8.8-9.2 \mathrm{GeV}$ there is a deconfinement PT, it means that for the first time we have an experimental evidence that QCD may have a tricritical endpoint instead of a critical one.

In our opinion it is remarkable that an existence of two phase transitions in QCD is also supported by the most successful transport approach developed in Giessen [40, 41]. It is a well-known PartonHadron-String Dynamics transport model. Moreover, it seems that this approach predicts the CSR PT of first order at the collision energy about $\sqrt{s}_{N N} \simeq 4 \mathrm{GeV}$, while the deconfinement PT of second order is predicted at about $\sqrt{s}_{N N} \simeq 10 \mathrm{GeV}[40,41]$. In contrast to the PT signals presented above, the transport model of Refs. [40, 41] demonstrates two cross-overs due to the finite size of the system.

It is possible that the CSR is responsible for the decrease of the inverse slope parameter of $\mathrm{K}^{+}$ mesons, when collision energy increases from $\sqrt{s}_{N N}=4.3 \mathrm{GeV}$ to $\sqrt{s}_{N N}=4.9 \mathrm{GeV}$. As one can see from the two topmost triangles in Fig. 4 the inverse slope $T^{*}$, indeed, decreases. Of course, this maybe 


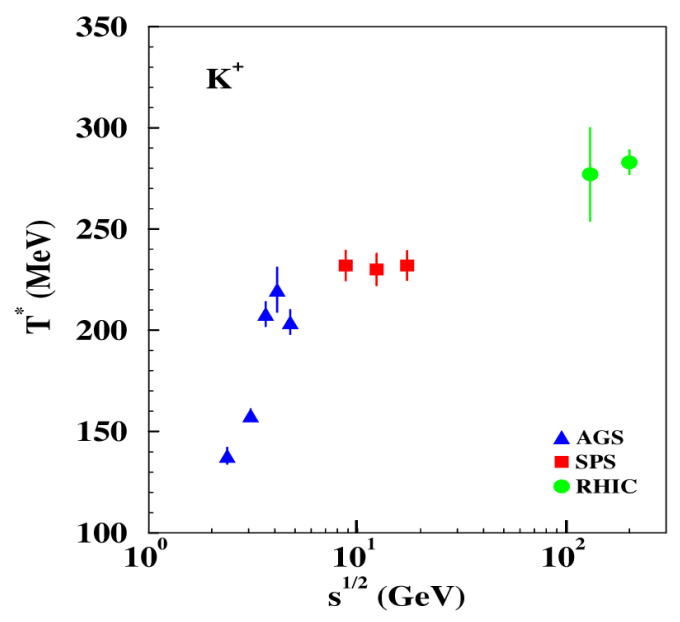

Figure 4. The collision energy dependence of the inverse slope parameter of $K^{+}$mesons. The two topmost AGS points (triangles) demonstrate the irregular behavior which may be related to the CSR PT. This plot is taken from Ref. [4].

a coincidence, but on the other hand this can be also a manifestation of the CSR. For the transverse momentum spectra of particles of mass $m_{k}$ which have the mean transverse hydrodynamic velocity $\bar{v}_{T}$ and temperature $T$ one can get the formula [42]

$$
T_{k}^{*}\left(p_{T} \rightarrow 0\right)=\frac{T}{1-\frac{1}{2} \bar{v}_{T}^{2}\left(m_{k} / T-1\right)} \approx T+\frac{1}{2} m_{k} \bar{v}_{T}^{2},
$$

where $p_{T}$ is the transverse momentum of particle. Since it is hard to imagine that an increase of collision energy can lead to a decrease of the hadronization temperature $T$ or to a decrease of the mean transverse hydrodynamic velocity $\bar{v}_{T}$, then the only possible cause of the decrease of $T_{k}^{*}$ for $K^{+}$ mesons is that their mass is reduced. It is interesting that NA49 Collaboration also reported a similar change of the inverse slope parameter of $K^{-}$mesons, but at a slightly higher collision energy interval $\sqrt{s}_{N N}=4.9-6.3 \mathrm{GeV}$ (see the left panel of Fig. 5 in [5]). Therefore, in order to verify or to disprove our hypothesis it would be necessary to measure the inverse slope parameter of $K^{ \pm}$mesons (or their transverse masses) with high precision in the collision energy range $\sqrt{s}_{N N}=4.3-6.3 \mathrm{GeV}$.

\section{Conclusions}

From the discussions above one can unambiguously conclude that the IST EoS is perfectly suited to determine the hard-core radii of all hadrons from the hadronic multiplicities which will be measured in the future experiments on RHIC, NICA and FAIR. We hope that these experiments will help to verify the new signals of the CSR PT and the deconfinement PT outlined here, and to experimentally locate the tricritical endpoint of the QCD phase diagram.

\section{Acknowledgements}

The authors are thankful to D. B. Blaschke, R. A. Lacey, I. N. Mishustin and R. D. Pisarski for fruitful discussions and valuable comments. K.A.B., A.I.I., V.V.S. and G.M.Z. acknowledge the support of the National Academy 
of Sciences of Ukraine. The work of K.A.B. and L.V.B. was performed in the framework of COST Action CA15213 "Theory of hot matter and relativistic heavy-ion collisions" (THOR). K.A.B. is thankful to the COST Action CA15213 for a partial support. V.V.S. thanks the Fundação para a Ciência e Tecnologia (FCT), Portugal, for the financial support to make research at the Centro de Astrofísica e Gravitação (CENTRA), Instituto Superior Técnico, Universidade de Lisboa. L.V.B and K.A.B. thank the Norwegian Centre for International Cooperation in Education (SIU) for financial support under grant "CPEA-LT-2016/10094 - From Strong Interacting Matter to Dark Matter". The work of L.V.B., K.A.B. and E.E.Z. was also supported by the Norwegian Research Council (NFR) under grant No. 255253/F50 - "CERN Heavy Ion Theory". K.A.B. is very thankful to the organizers of the ICNFP2017 for the warm hospitality in OAC.

\section{References}

[1] J. Schukraft, QM2017: Status and Key open Questions in Ultra-Relativistic Heavy-Ion Physics, arXiv:1705.02646 [hep-ex] and references therein.

[2] M. Gazdzicki, Z. Phys. C 66, 659 (1995).

[3] M. Gazdzicki and M.I. Gorenstein, Acta Phys. Polon. B 30, 2705 (1999).

[4] M. I. Gorenstein, M. Gazdzicki and K. A. Bugaev, Phys. Lett. B 567, 175 (2003).

[5] S. Pulawski [NA61 Collaboration], PoS CPOD 2014 (2015) 010.

[6] M. Gazdzicki, M. I. Gorenstein and P. Seyboth, Acta Phys. Polon. B 42, 307 (2011).

[7] K. A. Bugaev, Acta. Phys. Polon. B36, 3083 (2005).

[8] K. A. Bugaev, Phys. Part. Nucl. 38, 447 (2007).

[9] K. A. Bugaev et al., Phys. Part. Nucl. Lett. 12, 238 (2015).

[10] K. A. Bugaev et al., Eur. Phys. J. A 52, 175 (2016).

[11] K. A. Bugaev et al., Eur. Phys. J. A 52, 227 (2016).

[12] K. A. Bugaev et al., arXiv:1709.05419 [hep-ph] (accepted to Phys. Part. Nucl. Lett.).

[13] K. A. Bugaev et al., arXiv:1711.07283 [nucl-th].

[14] K. A. Bugaev et al., Nucl. Phys. A 970, 133 (2018).

[15] V. V. Sagun et al., arXiv:1703.00049 [nucl-th].

[16] K. A. Bugaev, A. I. Ivanytskyi, V. V. Sagun, E. G. Nikonov and G. M. Zinovjev, arXiv:1704.06846 [nucl-th].

[17] V. V. Sagun, A. I. Ivanytskyi, K. A. Bugaev and I. N. Mishustin, Nucl. Phys. A 924, 24 (2014).

[18] A. Andronic, P. Braun-Munzinger and J. Stachel, Nucl. Phys. A 772, 167 (2006).

[19] D. R. Oliinychenko, K. A. Bugaev and A. S. Sorin, Ukr. J. Phys. 58, 211 (2013).

[20] K. A. Bugaev, D. R. Oliinychenko, A. S. Sorin, G. M. Zinovjev, Eur. Phys. J. A 49, 30 (2013).

[21] K. A. Bugaev et al., Europhys. Lett. 104, 22002 (2013).

[22] V. V. Sagun, Ukr. J Phys. 59, 755 (2014).

[23] V. V. Sagun et al., Ukr. J. Phys. 59, 1043 (2014).

[24] K. A. Bugaev et al., Ukr. J. Phys. 61, 659 (2016).

[25] R. Venugopalan and M. Prakash, Nucl. Phys. A 546, 718 (1992).

[26] L. M. Satarov, M. N. Dmitriev and I. N. Mishustin, Phys. Atom. Nucl. 72, 1390 (2009).

[27] K. A. Bugaev, Nucl. Phys. A 606, (1996) 559.

[28] K. A. Bugaev, Phys. Rev. Lett. 90, (2003) 252301 and references therein.

[29] R. Hagedorn, Nuovo Cim. Suppl. 3, 147 (1965).

[30] L. Adamczyk et al., [STAR Collaboration], Phys. Rev. C 93, (2016) 021903.

[31] P. Senger, Eur. Phys. J. A 52, 217 (2016) and references therein. 
[32] P. Senger, Nucl. Phys. A 862-863, 139 (2011) and references therein.

[33] J. Rafelski, Phys. Lett. B 62, 333 (1991).

[34] K. A. Bugaev, M. I. Gorenstein, B. Kämpfer and V. I. Zhdanov, Phys. Rev. D 40, 2903 (1989).

[35] K. A. Bugaev, M. I. Gorenstein, D. H. Rischke, Phys. Lett. B 255, 18 (1991).

[36] A. K. Wroblewski, Acta Phys. Pol. B 16, 379 (1985).

[37] L. G. Moretto, K. A. Bugaev, J. B. Elliott and L. Phair, Europhys. Lett. 76, 402 (2006).

[38] M. Beitel, K. Gallmeister and C. Greiner, Phys. Rev. C 90, 045203 (2014).

[39] M. Beitel, K. Gallmeister and C. Greiner, J. Phys. Conf. Ser. 668, 012057 (2016).

[40] W. Cassing, A. Palmese, P. Moreau and E. L. Bratkovskaya, Phys. Rev. C 93, 014902 (2016).

[41] A. Palmese et al., Phys. Rev. C 94, 044912 (2016).

[42] M. I. Gorenstein, K. A. Bugaev and M. Gazdzicki, Phys. Rev. Lett. 88, 132301 (2002) and references therein. 